\title{
Evaluation of biological debris on reusable endodontic instruments subjected to different cleaning methods prior to sterilization
}

\author{
Poorva Khullar', Deepak Raisingani², Shailendra Gupta ${ }^{2}$, Kundendu A Bishen² \\ 1. Department of Conservative Dentistry and Endodontics, \\ Mahatma Gandhi Dental College and Hospital, Jaipur, India \\ 2. Mahatma Gandhi Dental College and Hospital, \\ Mahatma Gandhi University of Medical Sciences and Technology Sitapura, Jaipur, India
}

doi: 10.3396/ijic.v9i2.015.13

\begin{abstract}
The aim of this study was to evaluate the presence of biological debris on reusable endodontic instruments subjected to different cleaning methods prior to sterilization. Sixty endodontic hand instruments (K-files \#15-40 used in 3-4 teeth for cleaning and shaping) were analysed and were divided into 3 groups on the basis of decontamination protocols used. Twenty new K-files were used as controls. The evaluation of the effectiveness of the cleaning methods used was based on the amount of residual debris detected by immersion of instruments in Van Gieson's solution for 3 minutes. The samples were then rinsed in distilled water and dried on endodontic stand and analyzed by light microscopy.
\end{abstract}

Residual biological debris was observed on $93 \%$ of all the samples taken. The mean value of Maximum Biologic Contamination (MBC) was $20 \%$ for the group where instruments were brushed manually and immersed in alcohol, $15 \%$ in the group in which commercially available disinfectant were used and $11 \%$ for the group where instruments were ultrasonically cleaned. There was statistically significant difference between the cleaning protocols applied $(\mathrm{P}<0.001)$.

The methods used to clean endodontic instruments appear to be generally ineffective for the removal of biological debris. The best method was the one that included mechanical, chemical and ultrasonic cleaning of the instruments.

\section{Key words}

Dental instruments and microbiology; Waste products and prevention and control; Infection control dental and methods

\section{Corresponding Author}

Poorva Khullar

Department of Conservative Dentistry and Endodontics, Mahatma Gandhi Dental College and Hospital, Jaipur, India Email: poorvakhullar13@gmail.com 


\section{Introduction}

Sterilization describes a process that destroys or eliminates all forms of microbial life and is carried out by physical or chemical methods. ${ }^{1}$ Disinfection describes a process that eliminates many or all pathogenic microorganisms, except bacterial spores, on inanimate objects. In health-care settings, objects usually are disinfected by liquid chemicals or wet pasteurization. ${ }^{1}$ Cross-infection is a major issue in the dental care setting because of concerns about transmission of disease via the oral cavity. Endodontic treatment may directly involve contact with saliva, blood and infected pulp tissue. The US Centers for Disease Control and Prevention (Centers for Disease Control 1987) emphasized that all blood and body fluids that have been implicated in transmitting bloodborne infections should be considered as potentially infectious, regardless of a patient's infectious status. According to Australian National Health and Medical Research Council (NHMRC) guidelines (2002), instruments used in invasive dental procedures (including root canal treatment) are considered to involve a critical site, and should be sterile at the time of use. ${ }^{2}$

During the cleaning and shaping of the root canal, residual organic and inorganic material accumulates on the working sections of endodontic instruments. The possibility of the transmission of these materials from one patient to another is substantial because they can act as antigens, infectious agents or non-specific irritants. $^{3}$

There has been very little evaluation of the efficacy of cleaning procedures used for contaminated endodontic files. Segall et al. examined the manual cleaning of files with gauze or sponges and found these cleaning procedures to be ineffective in producing completely clean files. However, the quantification methods used in this study were subjective and relatively inaccurate. ${ }^{4,5}$

Murgel et al. demonstrated no significant difference in the amount of manufacturing debris or biological debris found on used or unused files that had been cleaned in an ultrasonic bath. The authors also stated that none of the cleaning methods for the experimental groups consistently removed all of the biological debris. ${ }^{6}$
Smith et al. used a light microscope to examine files provided by general practitioners and a hospital dental clinic. Files received from the general practitioners were cleaned by hand-brushing and $76 \%$ of these files retained debris. Files received from the hospital dental clinic were cleaned in an ultrasonic bath and $14 \%$ of these files retained debris. There were no details of the number of times the instruments had been re-used and if the files were placed in a container or with other instruments in the ultrasonic bath.?

Endodontic files and reamers do not have internal surfaces that are inaccessible, but their construction and designs, which involve fluted and twisted sections, make both mechanical and chemical cleaning considerably more difficult. Consequently, residual biological debris may remain on the surface of the instrument even after sterilization. In this way, potentially infective material could be transmitted from an infected individual to other patients. Considerable attention has been drawn to the possibility of transmission of prions via contaminated instruments. ${ }^{3}$

There is increasing concern over the risk of iatrogenic transmission of Creutzfeldt-Jakob disease (CJD). The repeated use of dental instruments presents a theoretical risk for the development of infection because previous studies have shown that the trigeminal ganglia, the periodontal, gingival and pulpal tissues, and the tonsils could represent a significant level of infectivity in patients with CJD. ${ }^{3}$

The purpose of this study was to evaluate, using light microscopy, the presence of biological debris and the level of contamination on the surfaces of reusable endodontic instruments that were subjected to different cleaning methods prior to sterilization.

\section{Materials and methods}

The study involved stainless steel endodontic hand instruments (K-files \#15-40) which were used in approximately 3-4 teeth for cleaning and shaping of the canals. The instruments were discarded if they were visibly deformed or broken.

Sixty samples were divided into 3 groups for analysis. The first group included 20 samples, which after clinical use, were immersed in 3\% hydrogen peroxide, 
then brushed manually, immersed in $70 \%$ isopropyl alcohol and dried. The second group included 20 samples subjected to manual brushing, immersion in glutaraldehyde or any commercially available disinfectant, rinsing in water and drying. The third group included 20 samples that were brushed manually, soaked in $1 \%$ sodium hypochlorite, put into ultrasonic baths with disinfectant, rinsed in water and dried. The fourth group (control group) consisted of 20 samples, which were stained in Van Geison's stain solution immediately upon removal from their original packages, rinsed in distilled water and dried.

Van Gieson's staining method, which was used in this study, is the simplest method of obtaining a differential staining of collagen and other connective tissue.

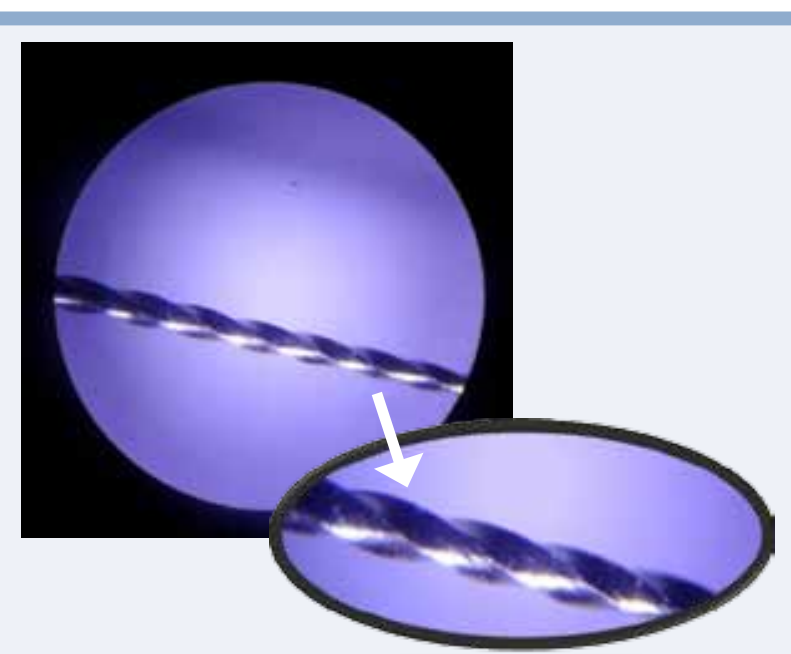

Figure 1. Showing clean surface without any debris (score 0 )

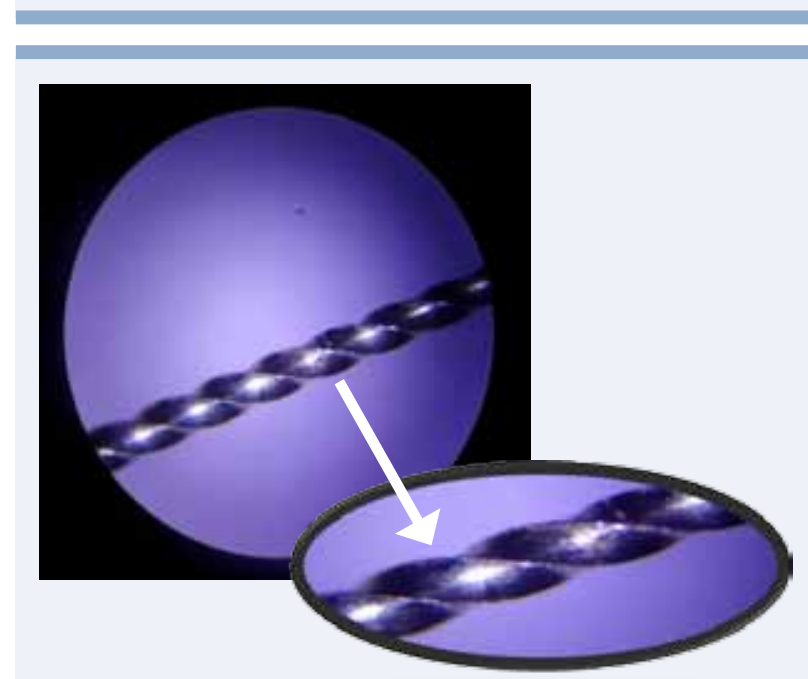

Figure 2. Showing organic film on the file surface (score 1)
Van Gieson's solution is a mixture of picric acid and acid fuchsin. It has been used in previous studies to demonstrate organic debris. ${ }^{2}$ The resulting pattern in dense structures, such as muscle tissue, erythrocytes, cytoplasm and fibrin, is a yellow stain, whereas more loosely composed tissues, such as collagen and reticulin, are stained red. ${ }^{3}$

According to the criteria specified by Linsuwanont et al., ${ }^{2}$ the residual debris was categorized as stained debris (red or orange aggregates on the surface of the instrument), organic film (a thin, red unstructured layer covering a part of the instrument), unstained debris (unstained fine particles) or a clean surface. Using the amount present as a basis, the residual debris was scored as 0 - clean surface without any debris (figure

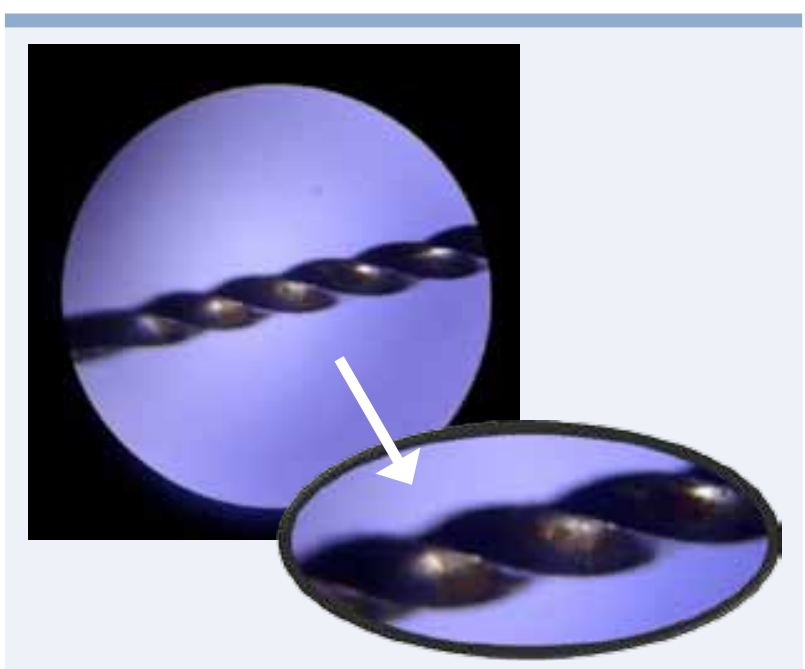

Figure 3. Showing single particles of debris scattered on the instrument surface (score 2)

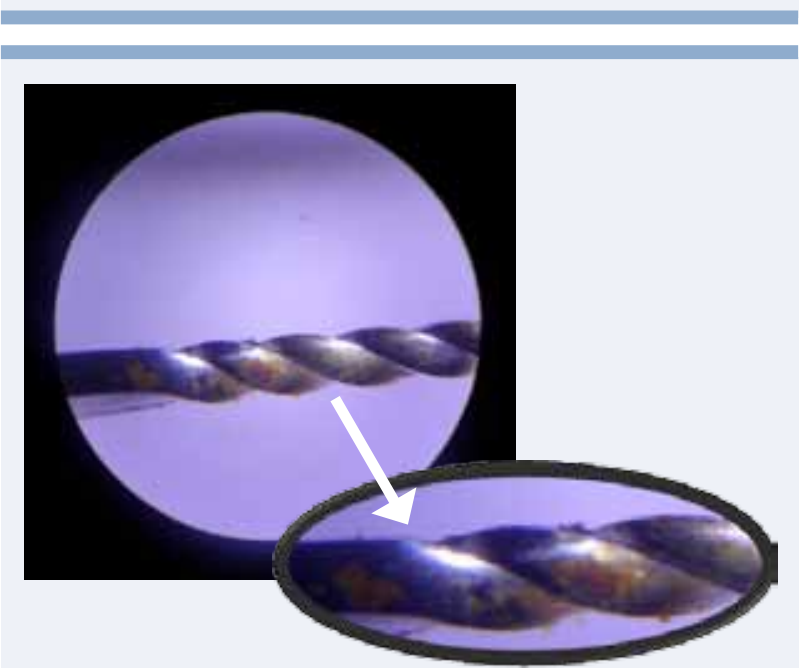

Figure 4. Organic particles covering the instrument surface as a continuous layer (score 3) 
1), 1- organic film (figure 2), 2- slight staining in the form of single particles of debris scattered on the instrument surface (figure 3), 3- moderate staining, organic particles covering the instrument surface as a continuous layer (figure 4), 4- a high level of staining, with the cutting flutes completely covered with debris.

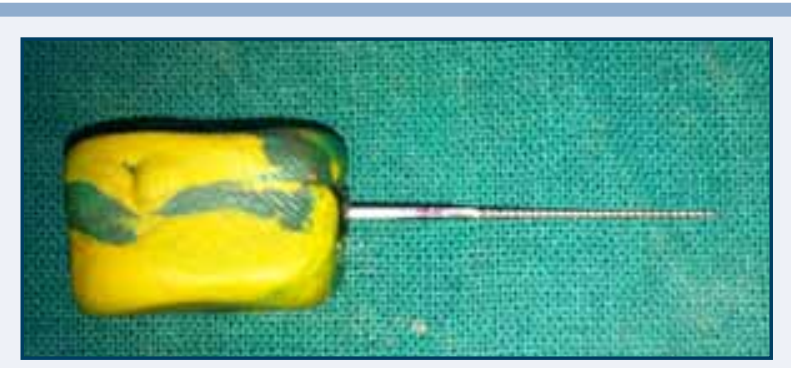

Figure 5. The rubber block designed for effective 90 degrees rotation of the file

The evaluation of the effectiveness of the cleaning methods used was based on the amount of residual debris detected by immersion of instruments in Van Gieson's solution for 3 minutes. The samples were then rinsed in distilled water and dried on endodontic stand. The endodontic instruments were analyzed under light microscope under 10x and 40x magnification to observe the stained organic material. The instruments were viewed on three levels: apical, middle and coronal. At each level, the samples were analyzed from four sides by sequential rotation through 90 degrees, which resulted in 12 measurements for each sample. A special rubber block was designed with a hole through which the handle of the instrument was placed to ensure stability of the instrument during microscopic examination (figure 5). The minimum value was 0 (no organic material present) and maximum was 48 (all surfaces were contaminated strongly with debris).

Statistical analysis was carried out using the nonparametric chi-squared test; a P-value of 0.001 was considered to be statistically significant.

\section{Results}

Careful examination under the light microscope revealed biological contamination on 56 (93.3\%) instruments. A moderate level of contamination (score 3) was registered on $18 \%$ of the tested instruments. A slight level of contamination (score 2) with scattered particles of organic debris was detected on 39\% of the instruments. The presence of an organic film (score 1) was observed on $37 \%$ of the instruments.
A clean surface (score 0 ) was observed on $6 \%$ of the instruments (figure 6).

There was a difference in the quality of cleaning of the instruments that depended on the protocol applied (alcohol, disinfectant or ultrasonic). The difference was statistically significant (chi-square 44.9 P value $<0.001$ ) with respect to the amount of residual debris (score 0-3) (Table I).

The analysis also showed that the first group had the highest value of MBC (Maximum Biologic Contamination), which was 33\% for instruments with score 3 and 4, 22\% for those with score 2 and $2 \%$ for instruments with score1. The mean value of MBC for the entire group was $20 \%$. There were no clean instruments in the group that was cleaned by manual brushing and alcohol. For the samples in the second group, the mean value of $\mathrm{MBC}$ for instruments with score 3 was $7 \%$; it was $32 \%$ for those with score 2 and $6 \%$ for instruments with score 1 . The mean value of MBC for the entire group was $15 \%$. The instruments in the third group, which received ultrasonic cleaning, showed a low level of contamination. The MBC value was $19 \%$ for instruments with score 1 , whereas the mean value of $\mathrm{MBC}$ for the entire group was $11 \%$ (Table II).

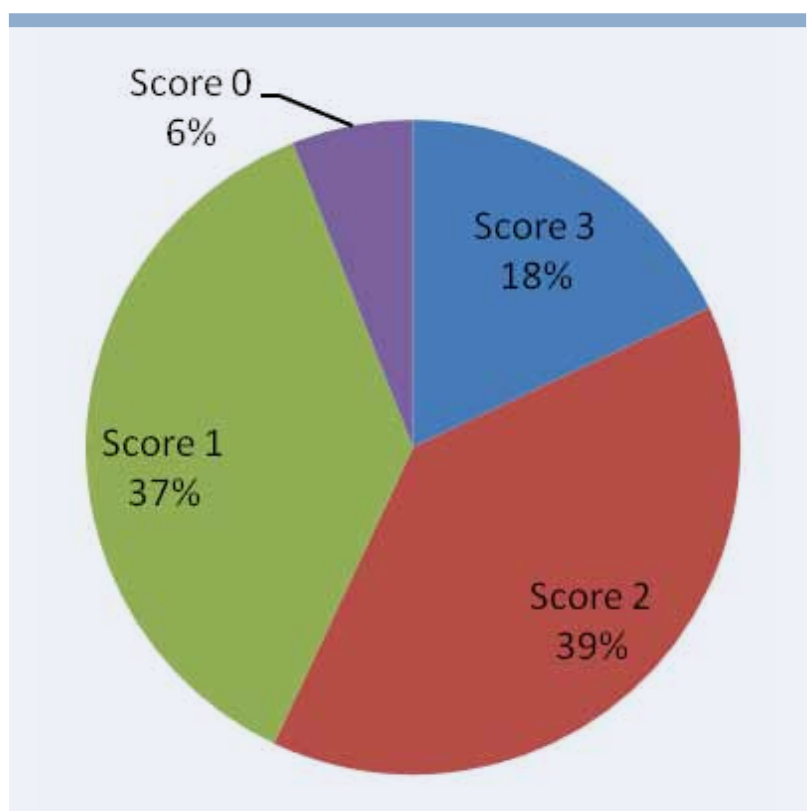

Figure 6. Level of contamination on tested instruments 
By testing the mean values of $M B C$, it was demonstrated that there was a statistically significant difference between the cleaning protocols applied (Chi-square $=$ 87.4, $\mathrm{P}$ value $<0.001$ ).

Microscopic analysis of the new instruments that were removed from the packaging of the manufacturer showed that all instruments had a certain amount of unstained metallic debris, but only one of the 20 instruments had stained particles on their surfaces.

\section{Discussion}

The results confirm those of earlier studies on the presence of residual debris on instruments that are used repeatedly in root canal treatment and show that contamination is possible after the reuse of instruments. ${ }^{8}$

Endodontic instruments, particularly files and reamers have been designated as single use instruments (SUI) in some dental jurisdictions. This action has arisen

Table I. Quantification of debris on endodontic hand instruments subjected to different cleaning methods

\begin{tabular}{rrrrrr} 
Cleaning Score & Protocol 1 & Protocol 2 & Protocol 3 & Chi square (df) & P value \\
\hline 3,4 & 9 & 2 & - & & \\
\hline 2 & 10 & 13 & - & $45(4)$ & $<0.001$ \\
\hline 0,1 & 2 & 5 & 20 & & \\
\hline
\end{tabular}

Table II. Maximum biological contamination with respect to the cleaning methods

\begin{tabular}{rrrrrr} 
Cleaning score & Protocol 1 & Protocol 2 & Protocol 3 & Chi square (df) & P value \\
\hline 3,4 & $27(33 \%)$ & $6(7 \%)$ & - & & \\
\hline 2 & $18(22 \%)$ & $26(32 \%)$ & - & $87.4(4)$ & $<0.001$ \\
\hline 0,1 & $2(2 \%)$ & $5(6 \%)$ & $20(11 \%)$ & & \\
\hline
\end{tabular}

out of concern for the difficulties encountered in their cleaning and sterilization after use and the possibility that they may act as vehicle for disease transmission when re-used. Of particular concern is the transmission of prion protein, a pathogenic isoform of a common host cell receptor, which causes acquired iatrogenic Creutzfeldt-Jakob disease (iCJD), a type of the spongiform encephalopathy. No treatment or prophylaxis is available for this disease and its acquisition nearly always proves fatal. ${ }^{9}$

At present there also is no universal standard for the preparation and sterilization of endodontic instruments prior to re-use. Most current methods fall short of consistently rendering instruments surgically sterile. Disease transmission associated with the reuse of endodontic hand and rotary files and reamers; however, has not proven itself to be a clinical problem in endodontic practice. Nonetheless, the threat of prion transmission via this route has become the basis for the decision to mandate that all such instruments be designated single use instruments (SUI) in some countries. $^{8}$

The UK Department of Health risk assessment for variant CJD (vCJD) has categorized dentistry as 'low risk' for the potential transmission of $\mathrm{V}_{\mathrm{cjd}} .^{8}{ }^{8}$ However, it is clear that infection is possible because endodontic interventions are frequent, endodontic instruments might come into direct contact with the pulpal and periodontal tissues and peripheral branches of the trigeminal nerve, and their inadequate cleaning might present a risk of transmission of infection. ${ }^{8}$

At present, Australian Standards recommend that endodontic files should demonstrate a 'macroscopic cleanliness' after a pre-sterilization cleaning procedure. 'Macroscopic cleanliness' of endodontic files can be difficult to assess due to their small size and fluted design. In addition, the accuracy of this assessment will vary with each individual. Adequate infection control protocols require a cleaning procedure that produces 
consistent and effective cleaning of endodontic files so that there is less reliance on subjective and inaccurate methods of assessment. ${ }^{4}$

Poor cleaning occurred particularly in the first two groups, in which the instruments were cleaned manually and immersed in alcohol (the first group) or commercially available disinfectants (the second group), and could be explained by the human factor, because the cleanness of each instrument depends largely on staff motivation and commitment.

The greatest amount of residual organic material was found on the instruments in the first group, which may be explained by the characteristics of alcohol, which dehydrates and fixates debris on the metal surface of the instrument. The presence of organic debris on the instruments in the second group may be explained by insufficient manual decontamination of the different types of endodontic instruments. ${ }^{3}$

Linsuwanont et al. and Murgel et al. examined individual decontamination techniques available in routine dental practice (mechanical cleaning with gauze and a sponge soaked in alcohol, cleaning with a brush, immersion in sodium hypochlorite, dry and wet storage of instruments and ultrasonic cleaning) and their combinations. They pointed out that the manual techniques for the removal of debris required considerable time, they were inefficient and carried with them a risk of introducing additional contamination. None of the decontamination techniques used in these studies was entirely efficient in removing debris., ${ }^{2,6}$

In the current study, the instruments in the third group were cleaned both manually and by an ultra-sound technique. The low values of MBC could be attributed to the effectiveness of ultrasonic waves in combination with manual cleaning and an efficient detergent. ${ }^{10}$

\section{Management of Infected Root canal Instruments}

The design of most root canal files and reamers makes their sterilization after use difficult. Most dental jurisdictions mandate that all endodontic instruments be sterilized prior to re-use. This includes (a) removal of all tissue and tissue by-products from the instrument surface and (b) sterilization by a device that destroys or inactivates all microorganisms and their by-products. Several sterilization protocols are currently in use; however, their effectiveness remains questionable. The most consistent and effective protocol appears to be one that includes hand and ultrasonic cleaning of instruments immediately after use, followed by processing in an autoclave that is constantly monitored to assure sterility. These methods appear to be effective in eliminating disease transmission caused by most microorganisms found within the root canal system but have been shown to be less effective in eliminating prion protein. However, based upon best current scientific evidence and the very low risk of prion transmission to patients during endodontic treatment in the USA and Canada, the Special Committee on Single Use Endodontic Instruments (SUI) feels that it is not currently warranted for clinicians to change the way in which they select endodontic files and reamers for re-use and sterilization. The Special Committee does recommend that practitioners prepare and sterilize instruments for re-use in accordance with "best evidence" currently available. It also encourages the development of new and innovative methods of sterilization that are simpler and are more efficient in eliminating the prion protein responsible for CJD. ${ }^{8,9}$

\section{Conclusion}

The methods for decontaminating endodontic instruments that are routinely applied in dental practices are generally ineffective in removing biological debris. Ultrasonic cleaning of endodontic files provides effective removal of biological debris. The goal of instrument sterilization in dentistry is to protect patients from cross contamination via instruments. Thus, utmost care should be taken to clean and sterilize each and every instrument before it is used in patients.

\section{Acknowledgements}

Department of Pathology (Mahatma Gandhi Medical College and Hospital) for provision of Von Geison stain.

\section{References}

1. Guideline for Disinfection and Sterilization in Healthcare Facilities, 2008

2. Linsuwanont $\mathrm{P}$, Parashos $\mathrm{P}$, Messer HH. Cleaning of rotary nickel-titanium endodontic instruments. Int Endod Journal 2004; 37: 19-28. http://dx.doi.org/10.1111/j.13652591.2004.00747.x 
3. Popovic J, Gasic J, Zivkovic S, Petrovic A, Radicevic G. Evaluation of biological debris on endodontic instruments after cleaning and sterilization procedures. Int Endod Journal 2010; 43: 336-341. http://dx.doi.org/10.1111/j.13652591.2010.01686.x

4. Van Eldik DA, Zilm PS, Rogers AH, Marin PD. A SEM evaluation of debris removal from endodontic files after cleaning and steam sterilization procedures. Australian Dental Journal 2004; 49(3): 128-135. http://dx.doi.org/10.1111/j.1834-7819.2004. tb00061.x

5. Segall RO, del Rio CE, Brady JM, Ayer WA. Evaluation of debridement techniques for endodontic instruments. Oral Surg Oral Med Oral Pathol 1977; 44: 786-791. http://dx.doi. org/10.1016/0030-4220(77)90388-7

6. Murgel CAF, Walton RE, Rittman B, Pecora JD. A comparison of techniques for cleaning endodontic files after usage: a quantitative scanning electron microscopic study. I Endod 1990; 16: 214-217. http://dx.doi.org/10.1016/S00992399(06)81672-9
7. Smith AJ, Dickson M, Aitken J, Bagg J. Contaminated dental instruments. J Hosp Infect 2002; 51: 233-235. http://dx.doi. org/10.1053/jhin.2002.1213

8. Letters S, Smith AJ, McHugh S, Bagg J. A study of visual and blood contamination onreprocessed endodontic files from general dental practice. British Dental Journal 2005; 199: 522 525. http://dx.doi.org/10.1038/sj.bdj.4812811

9. Joint AAE/CAE Special Committee on Single Use Endodontic Instruments (Dr. Gary Hartwell - Chair, Dr. Walter Bowles, Dr. Ove Peters, Dr. Marshall Peikoff and Dr. Calvin Torneck): American Association of Endodontics.

10. Muqbil I, Burke FJT, Miller CH, PalenikCJ. Antimicrobial activity of ultrasonic cleaners. Journal of Hospital Infection 2005; 60: 249-255. http://dx.doi.org/10.1016/j.jhin.2004.11.017 